\title{
A Case of an Intraorbital Wooden Chip Foreign Body Diagnosed and Removed Several Months after Injury Despite Repeated Imaging Study
}

\author{
Tetsutaro Oki ${ }^{a}$ b Nobutaka Shiotani ${ }^{a}$ Hiroshi Horiguchi ${ }^{a}$ Hisato Gunji ${ }^{a}$ \\ Tadashi Nakanoc \\ aDepartment of Ophthalmology, Kashiwa Hospital, The Jikei University School of Medicine, \\ Chiba, Japan; 'bepartment of Ophthalmology, Daisan Hospital, The Jikei University School \\ of Medicine, Tokyo, Japan; 'Department of Ophthalmology, The Jikei University School of \\ Medicine, Tokyo, Japan
}

\section{Keywords}

Intraorbital foreign bodies - Imaging · Inflammation · Delayed diagnosis · Orbit · Ocular trauma

\begin{abstract}
Intraorbital foreign bodies due to trauma are commonly encountered in clinical practice. However, organic foreign bodies, such as wood chips, can sometimes be difficult to diagnose. Here, we report the case of a patient with a wooden chip located intraorbitally that was not detected until spontaneous discharge, despite repeated diagnostic imaging. A 43-year-old intoxicated woman presented to our hospital with a bruised face and eyelid. The patient was diagnosed with conjunctivitis and iritis, but no foreign body was noted in repeated imaging studies since the first visit. Therefore, the patient was observed and managed conservatively. The patient returned to the hospital 114 days after the injury, with no improvement in her conjunctivitis. Examination revealed exposure of a foreign body in the lower eyelid conjunctiva, which was removed during emergency surgery. There are no clear criteria for imaging diagnosis of organic foreign bodies in the body. However, it may be possible to detect them by changing the imaging conditions and examining the computed tomography values in detail. In addition, in case of persistent clinical symptoms, such as inflammation, the image examination should be reviewed. In eyelid trauma, the medical history is sometimes unknown, so it must be remembered that a detailed examination and, in some cases, clinical symptoms are important in making a diagnosis.
\end{abstract}




\section{Case Reports in Ophthalmology}

\section{Introduction}

Intraorbital foreign bodies are commonly encountered in clinical practice. However, their diagnosis is not easy [1-9]. The diagnosis is made through a comprehensive assessment of the medical history, clinical findings (ocular motility disorder and suppurative inflammation), and diagnostic imaging techniques, such as computed tomography (CT), magnetic resonance imaging (MRI), and echo imaging [1, 2, 7, 9]. Clinical experience may also be helpful in diagnosis. Furthermore, organic foreign bodies, such as wooden chips, can be a breeding ground for infections, and thus can be fatal. Therefore, early detection and removal of foreign bodies is recommended [7]. However, identification of foreign bodies is sometimes very difficult. Here, we report a case in which a piece of wood remained undetected in the orbit for several months despite repeated examination by the ophthalmologist and radiologist, until some of the fragments were spontaneously exposed.

\section{Case Report}

A 43-year-old intoxicated woman bruised her left eyelid on a bar table after a fall and visited the emergency department of our hospital. Clinical examination revealed proptosis of the left eyeball, swelling of the eyelids, ocular motility disorder, and iritis. At the first visit to our hospital, 1 day after the onset of symptoms, the best-corrected visual acuity was $20 / 25$ in the right eye and 20/400 in the left eye (LE). The intraocular pressure was $12 \mathrm{~mm}$ $\mathrm{Hg}$ in the right eye and $24 \mathrm{~mm} \mathrm{Hg}$ in the LE. The image findings at the initial examination are shown in Figure 1. CT and MRI were performed, and images were immediately interpreted by an image interpretation specialist. CT images showed swelling of the left eyelid consistent with the site of injury and a subperiosteal hematoma in the orbital wall. There was no increased brightness that would suggest the presence of metal fragments. We excluded the possibility of foreign bodies because air density was observed on the lateral aspect of the lateral rectus muscle and the bony wall. The MRI showed damage to the lateral rectus muscle, in addition to the hematoma and swelling, but no intraorbital foreign body. The patient's white blood cell count was slightly increased. There was no fever or sensation of heat at the swollen site that would indicate infection. The patient complained of mild ocular motility disorder. Therefore, we followed up with HESS charts, which showed mild impairment in the abduction of the LE (Fig. 2). Based on these examinations, we diagnosed ocular motility disorder due to damage to the lateral rectus muscle and inflammation of the surrounding connective tissues. Furthermore, slit-lamp examination showed few anterior chamber inflammatory cells, traumatic mydriasis, and conjunctivitis. The patient was prescribed analgesics, antibacterial eye drops, steroid eye drops, and intraocular-pressurelowering eye drops. Ten days after the injury, visual acuity in LE (best-corrected visual acuity) had recovered to $20 / 25$, and diplopia disappeared. A repeat CT showed that compared to the day after the injury, the hematoma had dissipated, but fluid and air retention persisted. Compared with these findings, MRI examinations performed at 20 and 42 days after the injury also showed improvement in the injury, and no foreign body was detected in the orbit.

The patient returned 114 days after the injury with a complaint of prolonged hyperemia despite the use of eye drops. Slit-lamp examination showed conjunctivitis and an object covered by a membrane at the upper lateral corner of the LE (Fig. 3). The exposed object was mobile, and an attempt to grasp it with forceps resulted in bleeding from the periphery of the capsule. We suspected that a foreign body had strayed into the orbit at the time of injury and was spontaneously expelled over time. An updated CT of the orbit revealed a






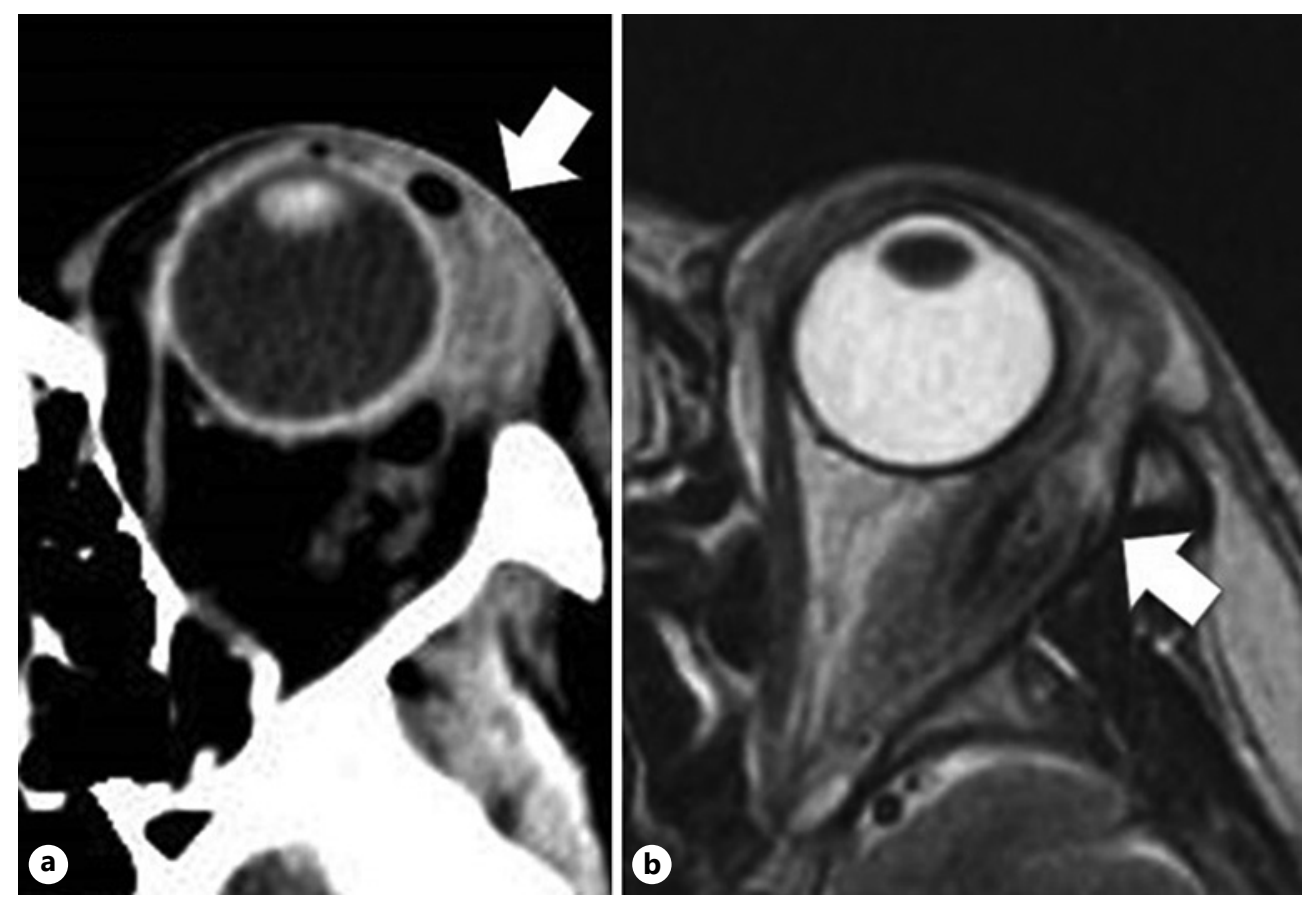

Fig. 1. Image findings. a Shows the CT image. The radiologist's report indicated that infection and foreign matter could not be identified. The arrow indicates a hematoma. Edema of the left orbit and eyelid is noted. Air density is found between the lateral aspect of the lateral rectus muscle and the bony wall. $\mathbf{b}$ Shows the MRI image. The radiologist's report suspected subperiosteal hematoma of the lateral wall of the left orbit and damage or swelling of the lateral rectus muscle (arrow).

foreign body of approximately $15 \mathrm{~mm}$ in the orbit. A total of nine wooden foreign bodies (maximal length $20 \mathrm{~mm}$ ) were removed during emergency surgery (Fig. 4). The postoperative course was uneventful. At the 2-month follow-up after surgery, the conjunctivitis improved remarkably. No foreign body was found on the postoperative CT, and there was no spontaneous discharge.

\section{Discussion}

We encountered a case of spontaneous expulsion of an intraorbital foreign body from the conjunctival sac a few months after an injury. Despite repeated imaging studies during follow-up, no foreign body was detected until it was spontaneously discharged. Although the spontaneous excretion of organic foreign bodies retained in the orbit several months to several years after eyelid trauma has been reported [3-5], to the best of our knowledge, no case of spontaneous expulsion from the conjunctival sac has been reported. Since organic foreign bodies left in the orbital space can cause visual dysfunction and serious infections in patients, prompt surgical removal after detection is recommended [7]. Fortunately, in our case, the patient had no serious complications, although the intraorbital foreign body was not detected despite five imaging procedures (two CTs and three MRIs) in 40 days. Previous reports have shown that CT and MRI are effective in detecting intraorbital foreign bodies [2,7-10]. Organic foreign bodies, such as wooden chips, are difficult to identify because wood chips contain carbon. Therefore, wooden chips may remain undetected in the orbit for a long time. Furthermore, the CT findings of retained wooden 


\section{Case Reports in Ophthalmology}

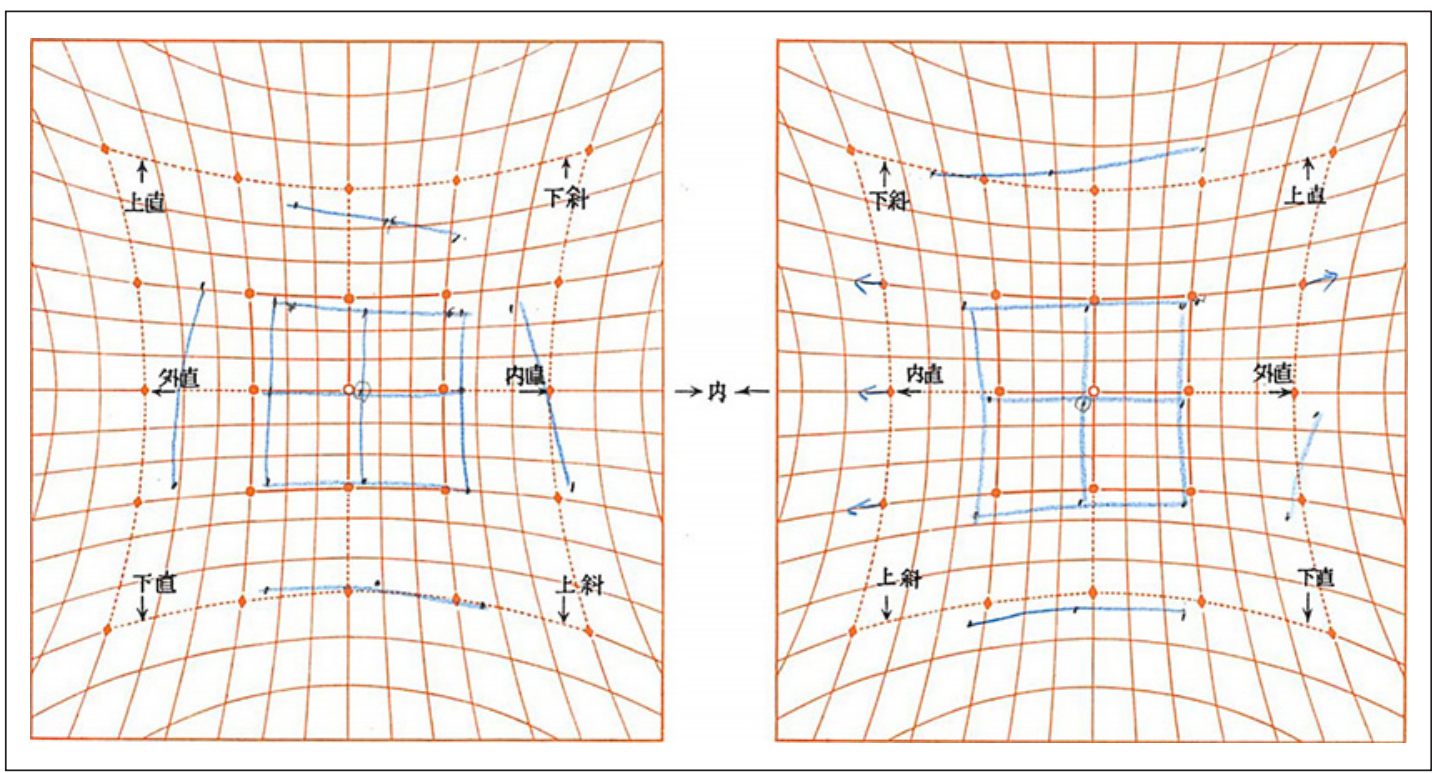

Fig. 2. HESS charts. The patient presented with supination and abduction deficits of the LE. It was thought to be due to the inflammation of the surrounding connective tissues.

Fig. 3. Photograph of the anterior segment slit, 114 days after the injury. Hyperemia of the bulbar conjunctiva and upper and lower eyelid conjunctiva is observed. A foreign substance wrapped in a membrane exposed from the lateral side (arrows) is visible with an open lower eyelid. Bleeding from the surroundings occurred when the area was touched.

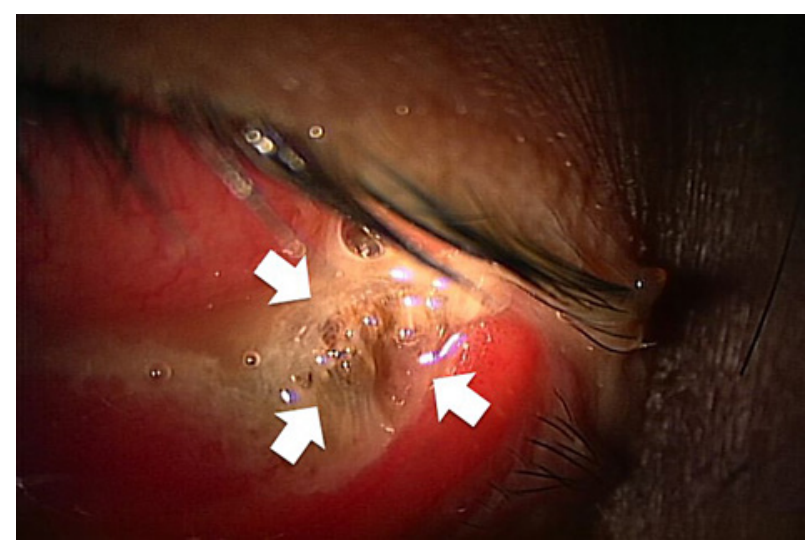

foreign bodies in the orbit differ greatly depending on the time of examination because their composition changes $[1,3,4,8,9]$. Specifically, wooden foreign bodies have a low density on CT in the early stages and tend to shift to a high density over time $[1,8$, $9,11]$.

We reviewed the CT images obtained in this case (Fig. 5). Air density was noted just below the bony wall on CT in the early stage of the injury. Therefore, we compared the air density with the CT values of the sinus cavity. The CT value of the sinus cavity was approximately $-1,000$ Hounsfield units (HU) (air density), whereas the CT value just below the bony wall was $-400 \mathrm{HU}$ (Fig. 5a: not air density). These findings revealed that what was assessed as air was not actually air. We further changed the window level of the image to air conditions to identify foreign bodies. Under air conditions at the window level, materials other than air appear white. Using this strategy, some foreign bodies were found in the air under the bony wall (Fig. 5b). We performed the same study on postoperative day $10 \mathrm{CT}$ images. The CT value of the area under the bony wall, which was thought to be air density, increased to $-240 \mathrm{HU}$. Previous studies reported that CT values of wooden chips retained in tissues change due to 


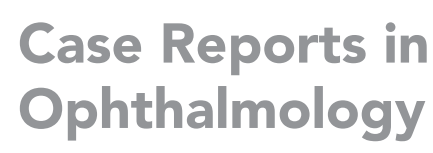

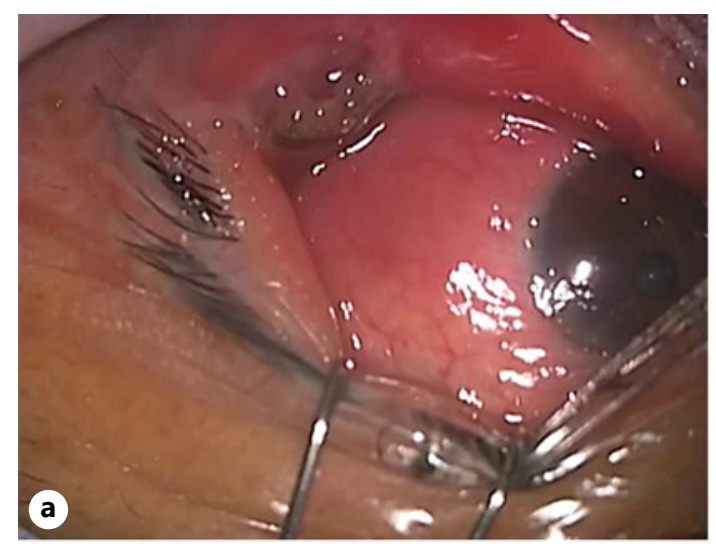


Fig. 4. Intraoperative findings and the removed foreign bodies. a Shows the exposed tip of a foreign object on the outside of the LE. The foreign matter is covered by a membrane. b Shows the removed foreign body caught in the forceps. c Shows a part of the removed foreign matter. The foreign body was cylindrical in shape, and a tissue similar to adipose tissue was attached to the inside. $\mathbf{d}$ Shows a total of nine wood chips that were removed from the patient. For comparison, the size of the sponge spear is $90 \mathrm{~mm}$. The longest foreign body was approximately $20 \mathrm{~mm}$ in length.

the absorption of tissue fluids and blood over a long period [1, 8, 9, 11]. In our case, CT values changed because of the absorption of tissue fluids by the wooden chips over 10 days and the presence of adipose tissue adhering to the wood chips. The MR images were also reviewed, but no evidence of foreign bodies could be detected.

In this case, we did not consider the possibility of foreign bodies at the initial examination because the patient's injury episode was unclear and there were no lacerations or other deep wounds on the eyelid that could allow a foreign body to enter the orbit. We neglected to change the CT conditions and examine the CT values in detail. Therefore, neither the ophthalmologist nor the radiologist was able to diagnose the foreign body until it was spontaneously discharged. Although there are no clear radiological criteria established for the diagnosis of organic foreign bodies in the orbit, clinical findings, such as persistent conjunctivitis, are as important as diagnostic imaging studies and are important signs that a review of the imaging findings may be indicated. Furthermore, when wooden chips and other foreign bodies are detected in the orbit, great care must be taken, as fragmentation at the time of removal can cause another infection in the orbit [12]. In our case, the wooden chips were already in several pieces at the time of removal. Thus, it was desirable to re-evaluate the patient postoperatively. Unfortunately, our patient did not return for follow-up 1 week after the surgery. 


\section{Case Reports in Ophthalmology}

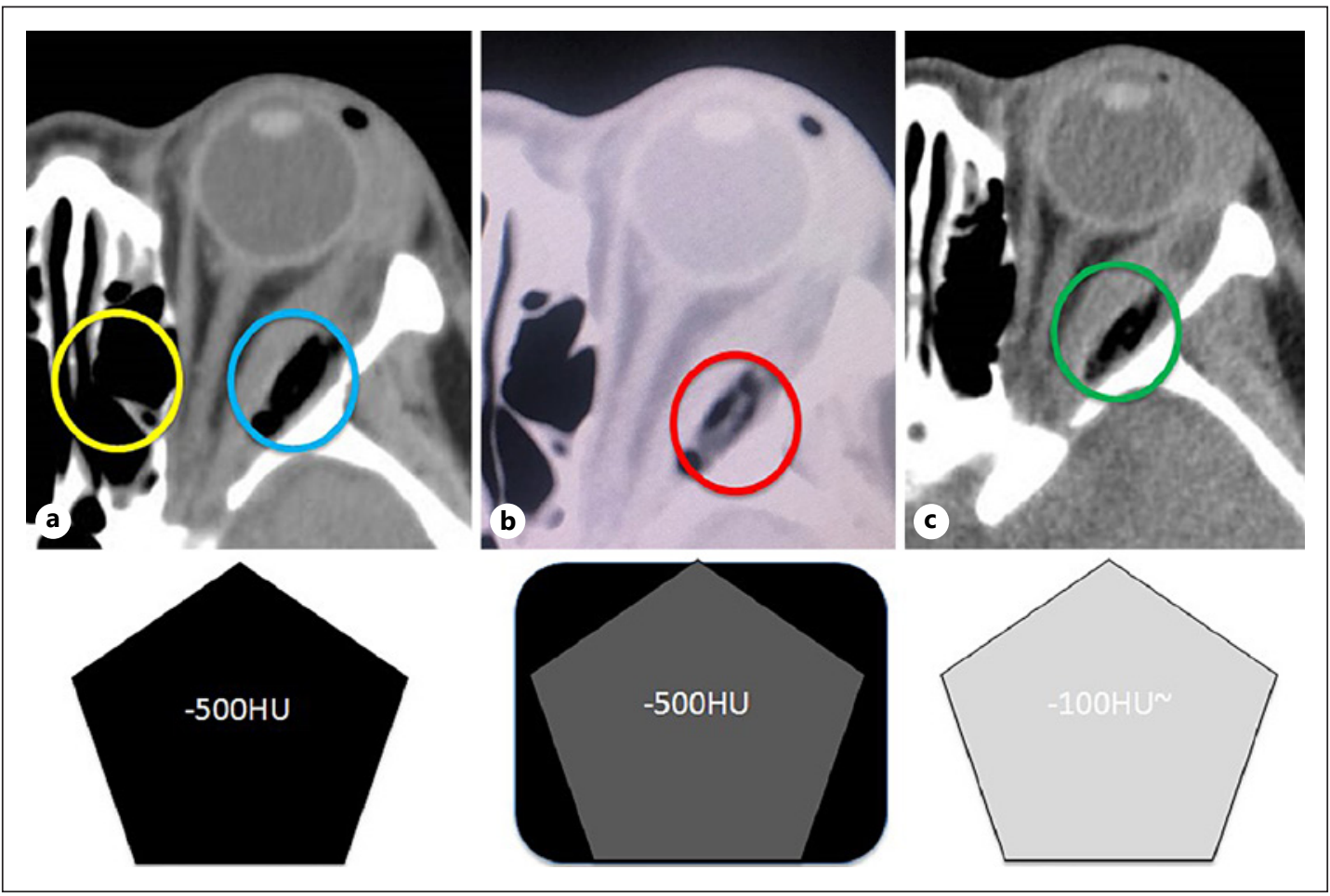

Fig. 5. Re-examination of the CT images. a Shows that the CT value of the sinus was $-1,000$ HU (yellow circle), while the CT value under the bony wall, originally thought to be air density was $-450 \mathrm{HU}$ (blue circle). The lower panel is an image of the CT value of a piece of wood $(-500 \mathrm{HU})$. b Shows that when the WL was changed to air level, materials other than air appeared white. An image emerged under the bony wall, suggesting a foreign body (red circle). The figure shows an image of a piece of wood under air WL. c Shows the CT on day 10 of the injury. The CT value in the area thought to be air was -200 HU (green circle). It was thought that the piece of wood absorbed tissue fluids and blood, which lead to changes in the CT value. Figure below shows the CT values of wood chips in the body approach the CT values of soft tissues over time. WL, window level.

In conclusion, we described the case of a patient with delayed diagnosis, despite repeated imaging examinations, and the spontaneous expulsion of an intraorbital foreign body from the conjunctival sac a few months after the injury. Our case indicated that clinical findings and imaging studies are both important for identifying intraorbital foreign bodies in cases of trauma to the eye.

\section{Acknowledgments}

We thank the patient for participating in this study.

\section{Statement of Ethics}

The protocol adhered to the tenets of the Declaration of Helsinki, and written informed consent was obtained from the patient for publication of this case report and any accompanying images. The paper is exempt from Ethical Committee approval because our institute (the Institutional Review Boards of The Jikei University) suggests single case report does not apply to that. 


\section{Conflict of Interest Statement}

The authors declare no competing financial interest.

\section{Funding Sources}

This research did not receive any specific grant from funding agencies in the public, commercial, or not-for-profit sectors.

\section{Author Contributions}

T.O., N.S., H.H., H.G., and T.N. contributed the conception and design of this case report. T.O. wrote the draft of this manuscript. T.O. and N.S. contributed to the diagnosis and treatment of the patient. All authors contributed to manuscript revision and approved the submitted final version.

\section{Data Availability Statement}

The imaging data are not publicly available due to their information that could compromise the patients' privacy.

\section{References}

1 Ho VT, McGuckin JF Jr, Smergel EM. Intraorbital wooden foreign body: CT and MR appearance. AJNR Am J Neuroradiol. 1996;17:134-6.

2 Green BF, Kraft SP, Carter KD, Buncic JR, Nerad JA, Armstrong D. Intraorbital wood. Detection by magnetic resonance imaging. Ophthalmology. 1990;97:608-11.

3 John SS, Rehman TA, John D, Raju RS. Missed diagnosis of a wooden intra-orbital foreign body. Indian J Ophthalmol. 2008;56:322-4.

4 Kim YH, Kim H, Yoon ES. Unrecognized intraorbital wooden foreign body. Arch Craniofac Surg. 2018;19:300-3.

5 Yoshii M, Enoki T, Mizukawa A, Okisaka S. Intraorbital wooden foreign body. Acta Ophthalmol Scand. 2004; 82:492-3.

6 Dunn IF, Kim DH, Rubin PA, Blinder R, Gates J, Golby AJ. Orbitocranial wooden foreign body: a pre-, intra-, and postoperative chronicle: case report. Neurosurgery. 2009;65:E383-4; discussion E384.

7 Fulcher TP, McNab AA, Sullivan TJ. Clinical features and management of intraorbital foreign bodies. Ophthalmology. 2002;109:494-500.

8 Roberts CF, Leehey PJ 3rd. Intraorbital wood foreign body mimicking air at CT. Radiology. 1992;185:507-8.

9 Boncoeur-Martel MP, Adenis JP, Rulfi JY, Robert PY, Dupuy JP, Maubon A. CT appearances of chronically retained wooden intraorbital foreign bodies. Neuroradiology. 2001;43:165-8.

10 Nagae LM, Katowitz WR, Bilaniuk LT, Anninger WV, Pollock AN. Radiological detection of intraorbital wooden foreign bodies. Pediatr Emerg Care. 2011;27:895-6.

11 Yago K, Suzuki M, Sasaki S, Nakamura Y. Wooden foreign body in the orbit. Jpn J Clin Ophthalmol. 1990;44:439-41.

12 Gonullu ME, Filinte GT, Cardak NG, Kucuk S, Akoz T. The surgical strategy for the intraorbital foreign bodies. J Craniofac Surg. 2016;27:1785-8.

\section{Karger'}

